\title{
Different Effects of 7-nitroindazole and L-NAME Administered Both Individually and Together on the Cardiovascular System of the Rat
}

\author{
F. KRISTEK ${ }^{1}$, M. DROBNA ${ }^{1}$, S. CACANYIOVA ${ }^{1}$ \\ ${ }^{1}$ Institute of Normal and Pathological Physiology, Centre of Excellence for Examination of \\ Regulatory Role of Nitric Oxide in Civilisation Diseases, Slovak Academy of Sciences, Bratislava, \\ Slovak Republic
}

Received March 18, 2014

Accepted May 30, 2014

On-line September 5, 2014

\section{Summary}

We evaluated the effects of $\mathrm{N}^{\mathrm{G}}$-nitro-L-arginine methylester (L-NAME) (50 mg/kg/day) and 7-nitroindazole (7NI) $(10 \mathrm{mg} / \mathrm{kg} /$ day $)$ administered from 10 th-16th week of age either individually or together on cardiovascular system of Wistar rats and SHR. Systolic blood pressure (SBP) was measured weekly by the plethysmographic method. For morphological studies, the animals $(n=10)$ were perfused with a fixative $(120 \mathrm{~mm} \mathrm{Hg})$, and thoracic aorta and carotid and coronary arteries were processed for electron microscopy. For functional investigation $(n=10)$, aortic rings were used in an organ bath. In Wistar rats, L-NAME evoked an increase of SBP; hypertrophy of the heart and arterial walls; an increase in cross-sectional areas (CSA) of endothelial cells (EC), muscle cells (SMC), extracellular matrix (ECM), and a decrease in acetylcholine-induced endothelial-dependent relaxation (EDR). 7NI evoked SBP-independent hypotrophy of the heart and arterial walls, a decrease in CSA of EC and SMC without affecting the CSA of ECM, and a mild decrease in acetylcholine-induced EDR. 7NI and L-NAME administered together evoked lower effect on BP and trophicity of the heart and all arteries, and a similar decrease in acetylcholine-induced EDR compared to L-NAME alone. In SHR, 7NI did not evoke any effect on the studied parameters.

\section{Key words}

Conduit arteries • Function • NO-deficiency • Structure

\section{Corresponding author}

F. Kristek, Institute of Normal and Pathological Physiology, Sienkiewiczova 1, 81371 Bratislava, Slovak Republic. Fax: +421 2 52868516. E-mail: Frantisek.kristek@savba.sk

\section{Introduction}

Changes in endogenous NO production lead to structural and functional alterations in the cardiovascular system (Kristek 2011). A long-term deficit in NO production due to administration of L-NAME (a nonspecific inhibitor of NO synthases) evokes a sustained increase in blood pressure accompanied with hypertrophy of the heart, pronounced hypertrophy of the arterial walls, decreased endothelium dependent relaxation (EDR) and increased contractility of conduit and resistant arteries. Similar cardiovascular alterations are observed in adult spontaneously hypertensive rats (SHR). Structural analysis of the carotid wall (tunica intima and media) in both adult SHR and Wistar rats treated from 10-16 weeks of age with N-G-nitro-Larginine methyl ester (L-NAME) revealed hypertrophy of endothelial and muscle cells and increased mass of the extracellular matrix (ECM) (Kristek et al. 1996). In adult Wistar rats treated with L-NAME, the structural and functional alterations in cardiovascular system mentioned above were blunted or prevented with administration of L-NAME along with exogenous NO donors (Kristek 2000, Torok and Kristek 2002). In contrast, similar administration of NO donors to adult SHR did not evoke any beneficial effect in this respect (Kristek et al. 2003). These data suggest that NO deficiency is likely not the main cause of cardiovascular alterations and that essential hypertension (represented by SHR) and NO-deficient hypertension are likely the result of different mechanisms. 
Contrary to the effects of L-NAME treatment, the long-term administration of the specific inhibitor of neuronal NO synthase (nNOS) 7-nitroindazole (7NI) evokes ambiguous effects on the cardiovascular system in normotensive rats. The majority of investigators did not observe alterations in blood pressure after chronic (days to weeks) NO synthase inhibition with 7NI (Beierwaltes 1997). To the best of our knowledge, there are no data except ours indicating the long-term effects of 7NI on the structure of the cardiovascular system in Wistar rats. In our previous experiments, we observed blood pressureindependent hypotrophy of the heart and the arterial walls of some conduit arteries in adult Wistar rats treated with 7NI from 10 to 16 weeks of age (Kristek et al. 2009) and in Wistar rats treated with 7NI from the prenatal period (via mothers treated with 7NI during the pregnancy and nursing) to 10 weeks of age (Kristek et al. 2013). Analysis of the carotid walls (tunica intima and media) in adult Wistar rats treated with 7NI from 10-16 weeks of age revealed a decreased mass (cross sectional area) of smooth muscle and endothelial cells without affecting the mass of the ECM (Kristek et al. 2009). Functional investigation in vitro supported the morphological findings. The studies demonstrated that both the contractility of the thoracic aortic rings and the response of blood pressure to noradrenaline were attenuated (Cacanyiova et al. 2009). Structural analysis of the arterial walls in SHR after 7NI treatment was not conducted. These findings suggest that NO production via modulation of NO synthases represents an attractive target for the regulation of the structure and function of the cardiovascular system.

The aim of this study was to evaluate how two types of NO synthase inhibitors, L-NAME and 7NI, administered either alone or in combination, affect the function and structure of the cardiovascular system in Wistar rats and SHR.

\section{Materials and Methods}

All procedures followed the guidelines set out in the Guide for the Use of Laboratory Animals (Ethics Committee for Experimental Work, Slovak Academy of Sciences, 1995). The animals were housed at a temperature range of $22-24{ }^{\circ} \mathrm{C}$ under a $12 \mathrm{~h}$ light/dark cycle and were fed a regular pellet diet.

Ten-week-old rats were used in the study. The animals were divided randomly into six groups of 20 animals each: 1) Wistar rats, 2) Wistar rats receiving $7 \mathrm{NI}$ in the drinking water $(10 \mathrm{mg} / \mathrm{kg} / \mathrm{day}), 3)$ Wistar rats receiving $7 \mathrm{NI}$ in food pellets $(10 \mathrm{mg} / \mathrm{kg} / \mathrm{day})$ and L-NAME in the drinking water $(50 \mathrm{mg} / \mathrm{kg} / \mathrm{day})$, 4) Wistar rats receiving L-NAME in drinking water (50 mg/kg/day), 5) SHR, and 6) SHR receiving 7NI in the drinking water $(10 \mathrm{mg} / \mathrm{kg} / \mathrm{day})$. Since the administration of L-NAME to SHR evoked high mortality (Ono et al. 1995) and the long-term administration of 7NI did not evoke any significant effect on cardiovascular system of SHR we decided not to treat SHR with L-NAME and L-NAME with 7NI. The ingestion of 7NI and L-NAME was monitored daily by tracking the intake of water and food. In all groups, systolic blood pressure (sBP) was measured each week by the plethysmographic method on the tail artery of pre-warmed animals.

After 6 weeks of treatment, ten animals from each group were taken for morphological investigation and ten animals were taken for in vitro studies.

\section{Morphological study}

The animals were sacrificed by an overdose of anesthesia using Zoletil ( $0.3 \mathrm{ml} / 100 \mathrm{~g}$ b.w.) and xylazine $(0.1 \mathrm{ml} / 100 \mathrm{~g} \mathrm{b.w.})$, the chest was opened and the cardiovascular system was perfused at a constant pressure of $120 \mathrm{~mm} \mathrm{Hg}$ for $10 \mathrm{~min}$ via a cannula placed in the left ventricle. $300 \mathrm{mmol} / \mathrm{l}$ glutaraldehyde in $100 \mathrm{mmol} / \mathrm{l}$ phosphate buffer was used as a fixative. After perfusion, the hearts were excised and weighed. Three conduit arteries supplying representative areas were chosen. The middle part of the thoracic aorta and carotid artery (CA) and upper part of the septal branch of the left descending coronary artery (RS) were excised, cleaned, divided into four segments about $1 \mathrm{~mm}$ long and processed according to electron microscopy procedure (for details Kristek 2000). Briefly, three randomly selected blocks of each artery were cut perpendicularly to the longitudinal axis. The inner circumference and arterial wall thickness (tunica intima and tunica media) were measured using light microscopy. The arterial wall thickness (tunica intima and tunica media) was measured at points in about $45^{\circ}$ intervals around the circumference of the artery. The inner diameter and cross sectional area (tunica intima and tunica media) was calculated.

The volume densities (proportional representation) of smooth muscle and endothelial cells and extracellular matrix in the wall (tunica media and tunica intima) of coronary artery were estimated quantitatively by the point counting method according to Weibel et al. (1966). Using the volume densities the cross sectional areas (CSA) were calculated. 
In vitro study

The thoracic aorta was isolated, cleaned of connective tissue and cut into rings (3-4 mm in length). The rings were vertically fixed between two stainless steel wires - triangles in $20 \mathrm{ml}$ incubation organ bath with Krebs solutions of the following milimolar composition: $\mathrm{NaCl} 118 ; \mathrm{KC} 15$; $\mathrm{NaHCO}_{3} 25 ; \mathrm{MgSO}_{4} 1.2$; $\mathrm{KH}_{2} \mathrm{P}_{4}$ 1.2; $\mathrm{CaCl}_{2} 2.5$; glucose 11; ascorbic acid 1.1; $\mathrm{CaNa}_{2}$ EDTA 0.032 . The solution was oxygenated with $95 \%$ oxygen and $5 \%$ carbon dioxide and kept at $37{ }^{\circ} \mathrm{C}$. The upper wire triangles were connected to electromechanical transducers Sanborn FT 10 and potentiometric recorders (Labora) for recording of changes in isometric tension. The resting tension was adjusted to $10 \mathrm{mN}$ and applied to each ring. Subsequently, the preparations were allowed to equilibrate for 60-90 min until stress relaxation no longer occurred. The relaxant responses were followed on the rings precontracted with submaximal dose of phenylephrine $\left(10^{-5} \mathrm{~mol} / \mathrm{l}\right)$ to achieve a stabile plateau of contraction. Following the rings were exposed to cumulative doses of acetylcholine $\left(10^{-9}-10^{-5} \mathrm{~mol} / \mathrm{l}\right)$. The extent of relaxation of arterial rings was expressed as a percentage of the phenylephrine-induced contraction.

The data obtained were expressed as means \pm SEM. Anova and the Bonferroni test for unpaired variables were used to assess statistical significance. Results were considered as significant when $\mathrm{P}<0.05$.

\section{Results}

\section{Basic parameters}

The administration of 7NI to Wistar rats did not significantly affect the sBP throughout the entire course of treatment. The administration of L-NAME to Wistar rats increased the sBP after the first week, and the sBP was significantly increased until the end of the experiment. From the first week of treatment on, Wistar rats receiving both L-NAME and 7NI showed increased sBP compared with untreated Wistar rats but decreased sBP compared with L-NAME-treated rats. In SHR, no differences in SBP were observed between the treated and untreated rats. The time-course of sBP changes in all groups is shown in Figure 1.

At the end of the experiment, the heart weight/body weight ratio (HW/BW) was decreased in Wistar rats after treatment with $7 \mathrm{NI}$ but increased after L-NAME administration. No difference in HW/BW was observed in Wistar rats receiving L-NAME together with
7NI. No change in HW/BW was observed in either untreated SHR or SHR treated with 7NI (Fig. 2).

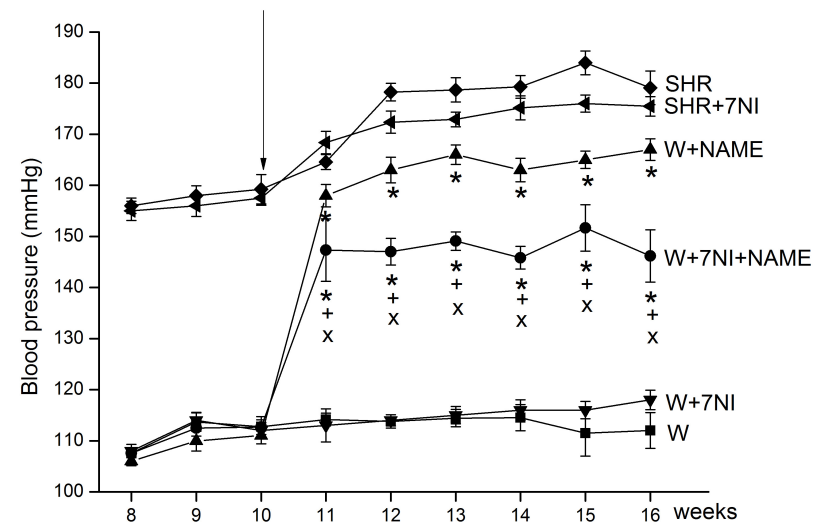

Fig. 1. Systolic blood pressure of Wistar rats (W), Wistar rats treated with 7-nitroindazole $(\mathrm{W}+7 \mathrm{NI})$, Wistar rats treated with $\mathrm{N}^{\mathrm{G}}$-nitro-L-arginine methylester (W+NAME), Wistar rats treated with 7NI along with L-NAME (W+7NI+NAME), spontaneously hypertensive rats (SHR), and SHR receiving 7NI (SHR+7NI). The values represent the data at the end of each week from 8th to 16 th weeks of age. Data are means \pm SEM. $* p<0.01$ vs. W; ${ }^{+} p<0.01$ vs. W+NAME; ${ }^{x} p<0.01$ vs. $W+7 N I$.

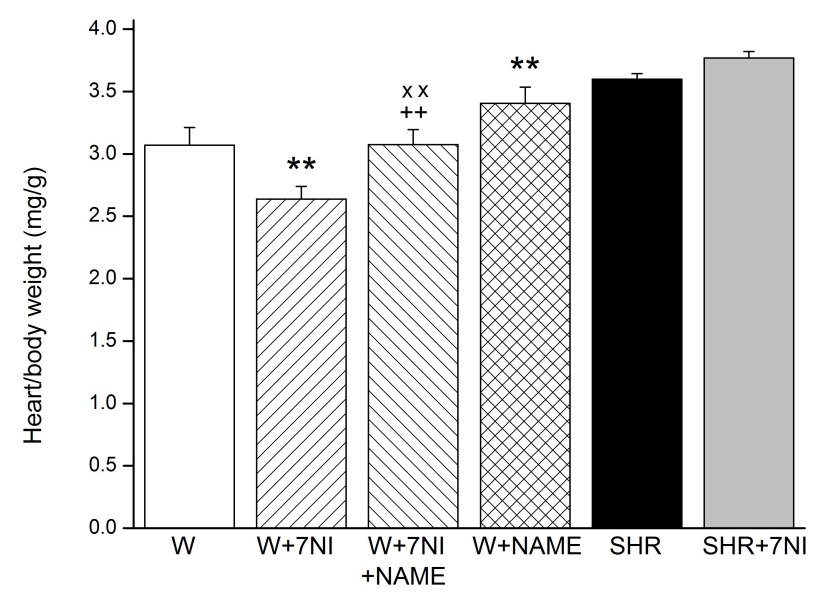

Fig. 2. Heart/body weight ratio $(\mathrm{mg} / \mathrm{g})$ of control Wistar rats (W), Wistar rats treated with 7-nitroindazole $(\mathrm{W}+7 \mathrm{NI})$, Wistar rats treated with $\mathrm{N}^{\mathrm{G}}$-nitro-L-arginine methylester (W+NAME), Wistar rats treated with 7NI along with L-NAME (W+7NI+NAME), spontaneously hypertensive rats (SHR), and SHR receiving 7NI $(\mathrm{SHR}+7 \mathrm{NI})$. Values represent the data at the end of the experiment. Data are means \pm SEM. ${ }^{* *} \mathrm{p}<0.01$ vs. $\mathrm{W}^{+{ }^{++}} \mathrm{p}<0.01$ vs. W+NAME; ${ }^{x \times} p<0.01$ vs. W+7NI.

\section{Geometry of the arteries}

At the end of the experiment, the CSA of the arterial wall (tunica intima and media) in all arteries of Wistar rats (thoracic aorta, carotid and coronary artery) was significantly decreased after 7NI treatment and significantly increased after L-NAME treatment. Concomitant administration of L-NAME and 7NI to 
Wistar rats resulted in an increase in the CSA of the arterial walls in comparison to the 7NI group and a decrease compared to L-NAME group. No effect was observed in all arteries of 7NI-treated SHR (Fig. 3A,B,C).
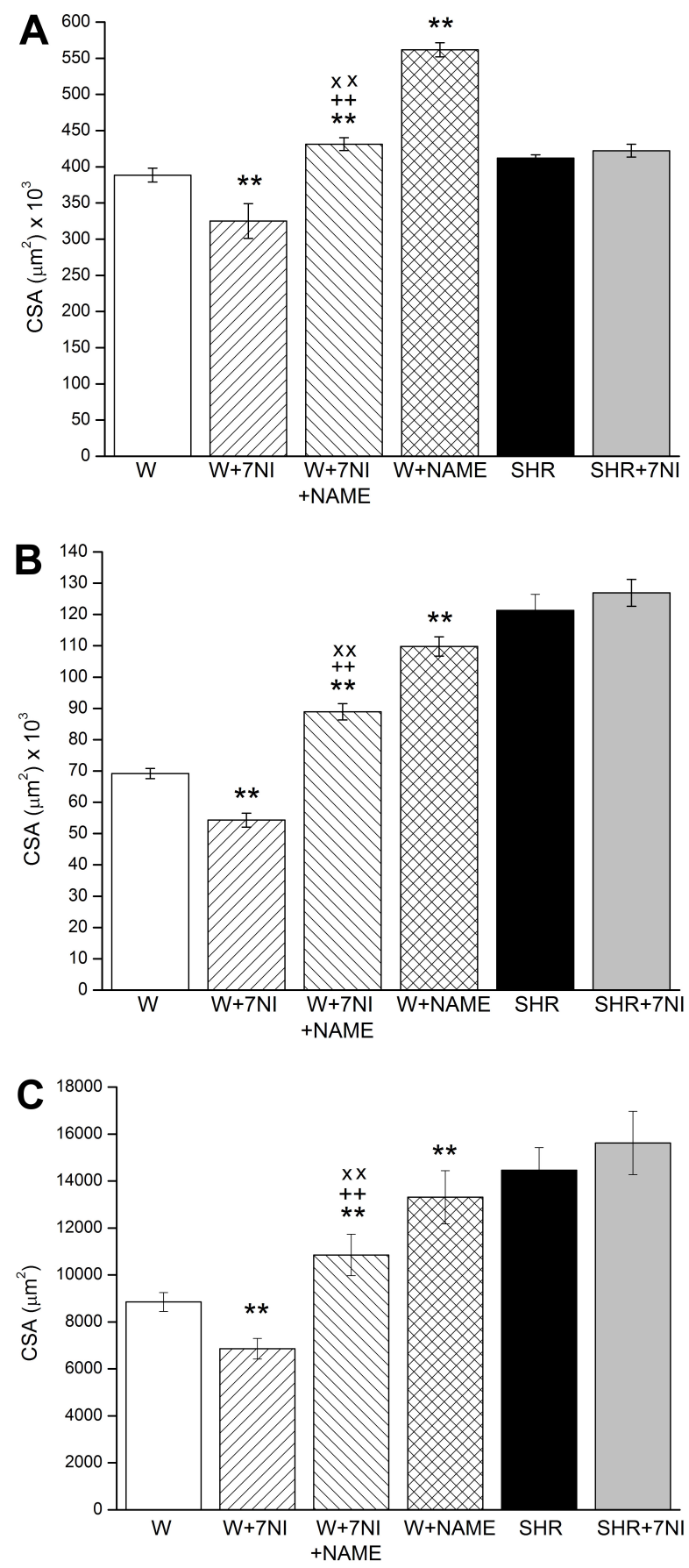

Fig. 3. Cross sectional areas of the thoracic aorta (A), carotid artery (B), and coronary artery (C) of control Wistar rats (W), Wistar rats treated with 7-nitroindazole $(\mathrm{W}+7 \mathrm{NI})$, Wistar rats treated with $\mathrm{N}^{\mathrm{G}}$-nitro-L-arginine methylester (W+NAME), Wistar rats treated with 7NI along with L-NAME (W+7NI+NAME), spontaneously hypertensive rats (SHR), and SHR receiving 7NI $(\mathrm{SHR}+7 \mathrm{NI})$. Data are means $\pm \mathrm{SEM} .{ }^{* *} \mathrm{p}<0.01$ vs. $\mathrm{W}^{++} \mathrm{p}<0.01$ vs. W+NAME; ${ }^{x x} p<0.01$ vs. $W+7 N I$.
Volume densities

The volume density (proportional representation) of smooth muscle cells in the coronary walls (tunica intima and media) of Wistar rats was decreased after $7 \mathrm{NI}$ treatment and L-NAME treatment. No differences in volume density were observed after the administration of L-NAME together with 7NI. The volume density of smooth muscle cells in SHR was not changed after 7NI administration (Fig. 4A).

The volume density of endothelial cells in the arterial walls did not change in Wistar rats after treatment with 7NI, L-NAME, and L-NAME + 7NI or in SHR after treatment with 7NI (Fig. 4A).

The volume density of the ECM was increased in Wistar rats after both 7NI treatment and L-NAME treatment, while no difference in the volume density was observed after treatment with 7NI together with L-NAME. In SHR, the administration of 7NI did not influence the volume density of the ECM (Fig. 4A).

\section{Cross-sectional areas}

The cross-sectional area represents the mass of a particular part of the arterial wall. In Wistar rats, the CSA of smooth muscle and endothelial cells was decreased after 7NI treatment and increased after L-NAME treatment. In Wistar rats, the concomitant administration of L-NAME with 7NI resulted in a decrease in the CSAs of smooth muscle and endothelial cells compared to L-NAME-treated rats. In Wistar rats treated with L-NAME and 7NI, the CSA of smooth muscle cells was increased, but no difference was observed in the CSA of endothelial cells, compared with untreated Wistar rats. In SHR, 7NI treatment did not affect the CSA of either smooth muscle or endothelial cells (Fig. 4B).

In Wistar rats treated with $7 \mathrm{NI}$, the CSA of the ECM was not changed in the arterial walls. Wistar rats treated with L-NAME exhibited an increase in the CSA of the ECM. The administration of both L-NAME and 7NI to Wistar rats significantly decreased the CSA of the ECM in comparison to L-NAME-treated rats and significantly increased the CSA of the ECM in comparison to untreated rats. No difference in the CSA of the ECM was observed between untreated SHR and SHR treated with 7NI (Fig. 4B).

\section{Functional study}

In Wistar rats, chronic treatment with $7 \mathrm{NI}$ did not affect the acetylcholine-induced concentration- 
dependent relaxation of the thoracic aorta pre-contracted by phenylephrine $\left(10^{-5} \mathrm{~mol} / \mathrm{l}\right)$. In comparison to untreated Wistar rats, administration of L-NAME alone and L-NAME together with 7NI significantly attenuated the acetylcholine-induced relaxation of the thoracic aorta. No difference in acetylcholine-induced relaxation was observed between untreated SHR and SHR treated with 7NI (Fig. 5).

\section{Discussion}

The main finding of this study is that $7 \mathrm{NI}$ in
Wistar rats, as opposed to L-NAME, induced blood pressure-independent hypotrophy of the heart and arterial walls of conduit arteries, decreased the mass of smooth muscle and endothelial cells without affecting the ECM, and did not decrease endothelium-dependent relaxation of the thoracic aorta. The administration of 7NI together with L-NAME moderated the cardiovascular alterations compared with the effects of L-NAME given alone. The administration of 7NI to SHR did not induce any significant effects in the majority of parameters studied.
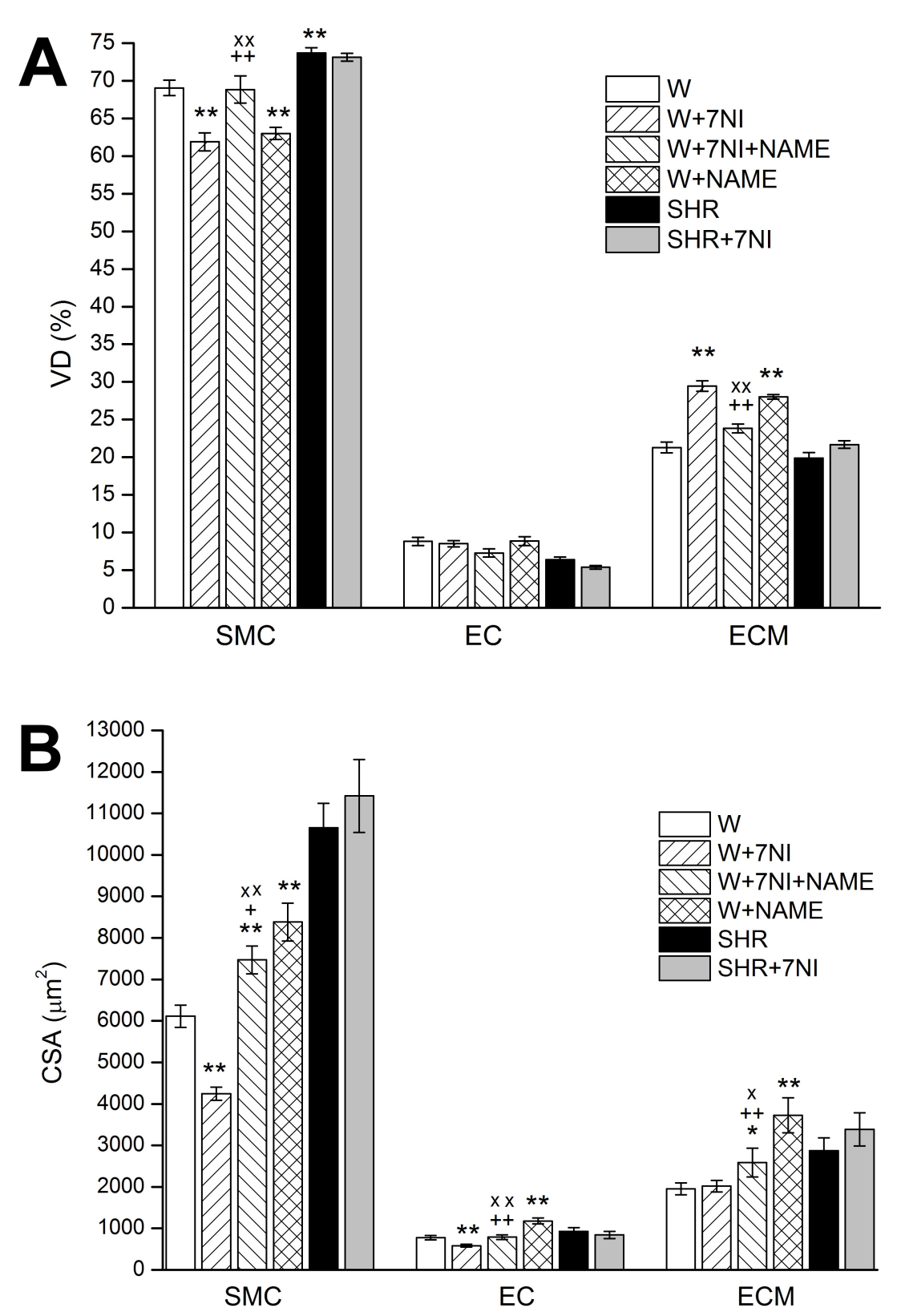

Fig. 4. Volume densities (percentual proportion) (A) and cross sectional areas (B), of smooth muscle cells (SMC), endothelial cells (EC), and extracellular matrix (ECM) in the wall (tunica intima+media) of coronary artery of control Wistar rats (W), Wistar rats treated with 7-nitroindazole $(\mathrm{W}+7 \mathrm{NI})$, Wistar rats treated with $\mathrm{N}^{\mathrm{G}}$-nitro-L-arginine methylester (W+NAME), Wistar rats treated with 7NI along with L-NAME (W+7NI+NAME), spontaneously hypertensive rats (SHR), and SHR receiving 7NI (SHR+7NI). Data are means \pm SEM. ${ }^{*} p<0.05, * * p<0.01$ vs. $W$; ${ }^{+} p<0.05,{ }^{++} p<0.01$ vs. W+NAME; ${ }^{x} p<0.05$, ${ }^{x \times} p<0.01$ vs. W+7NI. 


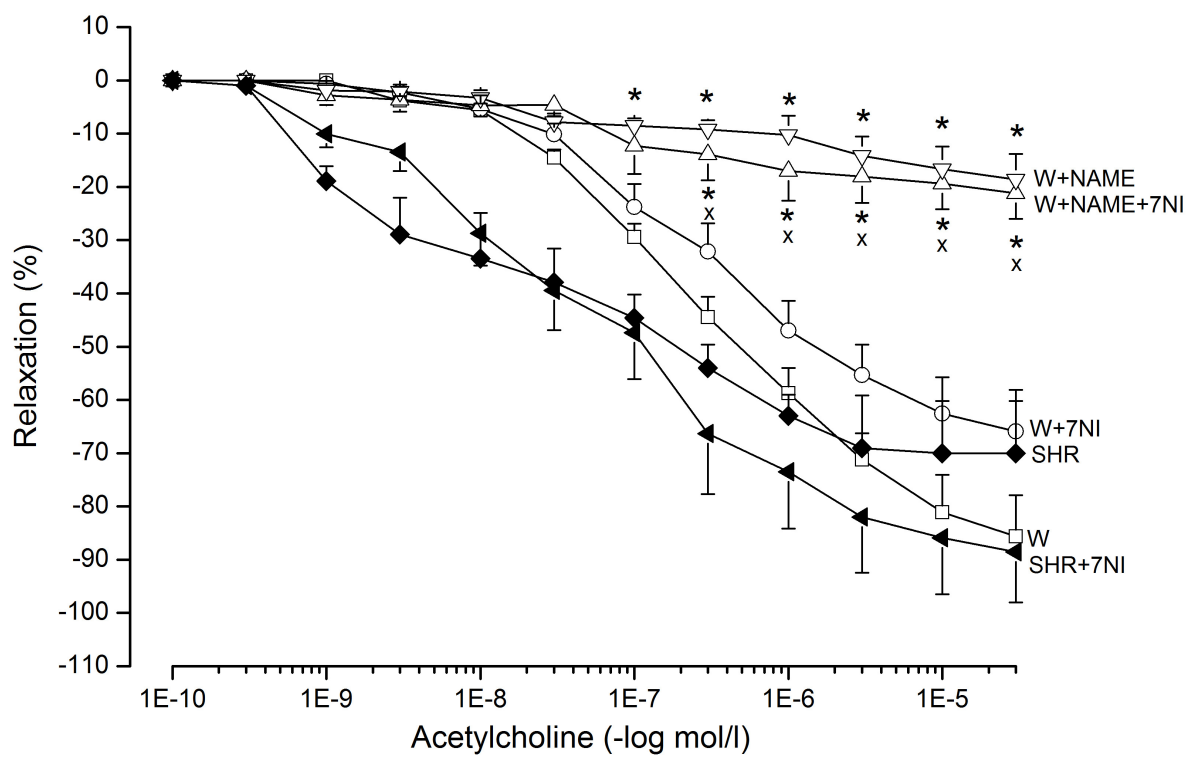

Fig. 5. Acetylcholine concentrationresponse curves of thoracic aorta isolated from control Wistar rats (W), Wistar rats treated with 7-nitroindazole $(\mathrm{W}+7 \mathrm{NI})$, Wistar rats treated with $\mathrm{N}^{G}$-nitro-L-arginine methylester (W+NAME), Wistar rats treated with 7NI along with L-NAME (W+7NI+NAME), spontaneously hypertensive rats (SHR), and SHR receiving $7 \mathrm{NI}(\mathrm{SHR}+7 \mathrm{NI})$. Values represent the data at the end of the experiment. Data are means \pm SEM. ${ }^{*} \mathrm{p}<0.01$ vs. $\mathrm{W} ;{ }^{\mathrm{x}} \mathrm{p}<0.01$ vs. $7 \mathrm{NI}$.

At the end of the experiment, sBP was increased by approximately $49 \%$ after L-NAME administration to Wistar rats. These data are in agreement with previous experiments, both from us and other groups, showing that long-term increases in blood pressure were induced in adult Wistar rats following treatment with various doses of L-NAME. These prior studies showed that the administration of L-NAME for 4-6 weeks at a dose of $40-50 \mathrm{mg} / \mathrm{kg}$ b.w./day increased the blood pressure by $40 \%$ (Sharma et al. 2012), $43 \%$ (Kristek and Gerova 1996), 47 \% (Bachhav et al. 2012), 49 \% (Kristek et al. 1996, Bernatova et al. 1999) and $63 \%$ (Felix et al. 2012). Treatment with L-NAME $(0.7 \mathrm{mg} / \mathrm{ml} /$ day $)$ for just 2 weeks increased the blood pressure by $38 \%$ (Toba et al. 2012).

As opposed to L-NAME, treatment with 7NI did not induce any significant effect on sBP. Throughout the entire experiment, the sBP was not significantly changed in either untreated Wistar rats or SHR. These data are in accordance with the observations of previous studies, where treatment with 7NI did not cause any significant change in the blood pressure of Wistar rats and SHR (Qadri et al. 1999, Cacanyiova et al. 2009, Kristek et al. 2009, Huang et al. 2012).

The administration of 7NI along with L-NAME resulted in SBP increases compared to untreated Wistar rats. By the end of the experiment, the SBP was increased by approximately $30 \%$. Compared to L-NAME-treated rats, the increase in SBP was significantly lower (by approximately $15 \%$ ). This effect was consistently observed throughout the entire experiment. These data suggest that $7 \mathrm{NI}$ blunts the potential of L-NAME to increase the BP of normotensive Wistar rats. To our best knowledge, this is the first report of such an effect.

Increases in BP are usually accompanied by cardiac hypertrophy. In agreement with this common observation, cardiac hypertrophy was observed in SHR and in Wistar rats treated with L-NAME alone. We and others have repeatedly observed cardiac hypertrophy after L-NAME administration to Wistar rats, even after a short time period (Kristek and Gerova 1996, Kristek et al. 1996, Bernatova et al. 1999, Nguelefack et al. 2009). Surprisingly, 7NI administration evoked an opposite, hypotrophic effect on the hearts of Wistar rats and no effect on the hearts of SHR. This effect was in agreement with our previous data (Kristek et al. 2009). In Wistar rats treated with $7 \mathrm{NI}$, the induction of cardiac hypotrophy without a decrease in blood pressure may be one of the events contributing to eventual heart failure, as the decreased mass of the myocardium would have to overcome the unchanged peripheral resistance. In SHR, $7 \mathrm{NI}$ did not evoke any significant effect on cardiac trophicity; while slight cardiac hypertrophy was observed, this effect was not significant. These data are consistent with the observations of Huang et al. (2012) and Cacanyiova et al. (2012), who did not observe cardiac hypertrophy after 7NI administration to SHR. The administration of 7NI along with L-NAME prevented both the hypertrophic effect of L-NAME and the hypotrophic effect of $7 \mathrm{NI}$. We suggest that the different effects of individually administered L-NAME and 7NI on the trophicity of the heart could explain why the joint administration of these inhibitors was not effective in altering the trophicity of the heart (as measured by the heart weight/body weight ratio). The data support the suggestion that NO deficiency due to L-NAME and/or 
7NI administration affects different regulatory pathways. Unfortunately, no prior studies are available to compare with our observations.

Hypertrophy of the walls of conduit arteries observed both in SHR and after long-term L-NAME administration to Wistar rats is in agreement with the majority of prior observations. As opposed to treatment with L-NAME, 7NI treatment causes hypotrophy of the arterial walls. The administration of both inhibitors together partially blunted their individual effects on trophicity in the arteries studied. The opposing effect of these inhibitors on the mass of the arterial walls in individual groups raised the question as to which component of the arterial walls was most affected and/or which component is responsible for these alterations. Analysis of the arterial walls (tunica intima and media) of the coronary artery revealed different volume densities (proportional representation) of smooth muscle cells, endothelial cells, and the ECM. The CSAs of individual arteries (representing $100 \%$ ) were markedly different in individual groups. Therefore, similar percentages of particular components represent different CSAs. Calculations based on volume densities revealed that $7 \mathrm{NI}$ treatment decreased the mass (CSA) of both smooth muscle and endothelial cells, leading to hypotrophy, while the mass of the ECM was unchanged. Similar data were observed in the carotid artery after 7NI treatment (Kristek et al. 2009). In L-NAME-treated Wistar rats, SHR and SHR treated with 7NI, an increase in the mass of endothelial and muscle cells as well as the ECM contributed to hypertrophy of the arterial walls (tunica intima and media). In rats treated with both $7 \mathrm{NI}$ and L-NAME, the mass of all components of the arterial wall was increased to a lower extent than in rats treated with L-NAME alone. Therefore, 7NI partially prevented the hypertrophic effect of L-NAME. No difference in this respect was observed between SHR and SHR treated with $7 \mathrm{NI}$.

The mechanisms behind the different responses in the cardiovascular system to L-NAME and 7NI treatment remain unclear, but many possibilities exist. $\mathrm{NO}$ is engaged in a variety of regulatory mechanisms, and changes to NO production may affect a cascade of multiple physiological and biochemical events. The question of which is the predominant stimulus remains unanswered. Decreased endothelium-dependent relaxation (EDR) in response to acetylcholine after L-NAME treatment suggests that L-NAME predominantly inhibits endothelial NO synthase (eNOS).
Preserved EDR after 7NI administration compared to untreated controls supports the suggestion that $7 \mathrm{NI}$ is a specific inhibitor of neuronal NO synthase (nNOS) activity, with no effect on eNOS (Moore et al. 1993, Beierwaltes 1995). nNOS is preferentially localized in nervous tissue. Because NO released from perivascular nerves blunts the sympathetic response by inhibiting neurogenic release (Rabelo et al. 2001, Hatanaka et al. 2006) and NO deficiency results in blood pressure increases, it seems unlikely that the inhibition of nNOS solely in nervous tissue (central or peripheral) can induce the observed alterations in the structure of the cardiovascular system in Wistar rats without affecting blood pressure. In addition to nervous tissue, the kidneys exhibited a high density of nNOS. NO synthesized in the macula densa is implicated in the regulation of renal vascular resistance, glomerular filtration, tubular reabsorption, tubuloglomerular feedback and renin secretion. nNOS inhibitors almost completely abolish the stimulation of renin secretion (He et al. 1995, Beierwaltes 1997, Paliege et al. 2004), thereby modulating angiotensin II production. An opposite (stimulatory) effect was observed in relation to the renin-angiotensinaldosterone system after eNOS inhibition (Usui et al. 2000, Linardi et al. 2004). Angiotensin II induces increases in blood pressure and strong proliferative and hypertrophic effects on the cardiovascular system (Naftilan 1992). We suggest that the different effects of L-NAME and 7NI treatment in relation to RAS could play an important role in their diverse consequences on the trophicity of the cardiovascular system in Wistar rats. The hypertensive effect of decreased NO production after 7NI treatment could be balanced by the down-regulation of angiotensin II. Milder cardiovascular alterations were observed in the group receiving 7NI together with L-NAME, compared with L-NAME alone, while greater alterations were observed compared to 7NI alone. These results are in agreement with our speculations.

As opposed to normotensive rats, only minor effects were observed in the cardiovascular system of SHR following 7NI treatment. In SHR, NO deficiency does not seem to be the main cause of pathological alterations in the cardiovascular system. Welch et al. (1999) showed that neither the expression of constitutive NO synthases nor the delivery of NO substrates and cofactors to the juxtaglomerular apparatus was decreased in SHR. We found that the long-term increase in the NO level due to NO donor administration did not prevent structural changes in conduit arteries in SHR, in contrast 
to the results for Wistar rats (Kristek et al. 2003). On the other hand, Welch et al. $(1999,2000)$ and Thorup and Persson (1996) observed diminished tubuloglomerular feedback in SHR. Tubuloglomerular overactivity has been ascribed to a diminished role for macula densa NO in buffering the vasoconstrictive response. We suggest that either this defect or insufficient regulation via $\mathrm{NO}$ synthesized locally by nNOS in the juxtaglomerular apparatus could be associated with the different structural and functional responses of the cardiovascular system of SHR compared to Wistar rats after $7 \mathrm{NI}$ administration.

In conclusion, the aforementioned results suggest that two different NO synthase inhibitors, L-NAME and 7NI, decrease the synthesis of the identical NO molecule yet induce different, and in many cases, opposite functional and structural effects on the cardiovascular system of Wistar rats. It seems likely that L-NAME and 7NI influence cardiovascular system of Wistar rats via different regulatory mechanisms. The relationships are more complicated by the fact that the roles for NO could be location dependent, varying based on different local requirements and conditions. Paradoxically, NO deficiency due to 7NI administration could induce a "protective" effect against the NO deficiency induced by the administration of L-NAME.

Further experiments are needed to unravel the varying roles of $\mathrm{NO}$ in the cardiovascular system and to determine the exact function of NO in physiological processes.

\section{Conflict of Interest}

There is no conflict of interest.

\section{Acknowledgements}

The study was supported by Ministry of Health of the Slovak Republic under the project registration number 2012/51-SAV-1 and grants VEGA 2/0067/14, 2/0074/14, Slovak Republic. The authors would like to thank L. Kosnacova for technical assistance.

\section{References}

BACHHAV SS, BHUTADA MS, PATIL SD, BASER B, CHAUDHARI KB: Effect of Viscum articulatum Burm. (Loranthaceae) in N-omega-nitro-L-arginine methyl ester induced hypertension and renal dysfunction. J Ethnopharm 142: 467-473, 2012.

BEIERWALTES WH: Selective neuronal nitric oxide synthase inhibition blocks furosemide-stimulated renin secretion in vivo. Am J Physiol 269: F134-F139, 1995.

BEIERWALTES WH: Macula densa stimulation of renin is reversed by selective inhibition of neuronal nitric oxide synthase. Am J Physiol Regul Integr Comp Physiol 272: R1359-R1364, 1997.

BERNATOVA I, PECHANOVA O, KRISTEK F: Mechanism of structural remodelling of the rat aorta during longterm NG-nitro-L-arginine methyl ester treatment. Jpn J Pharmacol 81: 99-106, 1999.

CACANYIOVA S, KRISTEK F, GEROVA M, KRENEK P, KLIMAS J: Effect of chronic nNOS inhibition on blood pressure, vasoactivity, and arterial wall structure in Wistar rats. Nitric Oxide 20: 304-310, 2009.

CACANYIOVA S, KRISTEK F, MALEKOVA M, ONDRIAS K: Effects of chronic neuronal nitric oxide-synthase inhibition on arterial function and structure in spontaneously hypertensive rats. J Physiol Pharmacol 63: 23-28, 2012.

FELIX AS, ROCHA VN, NASCIMENTO ALR, DE CARVALHO JJ: Carotid body remodelling in L-NAME-induced hypertension in the rat. J Comp Pathol 146: 348-356, 2012.

HATANAKA Y, HOBARA N, HONGHUA J, AKIYAMA S, NAWA H, KOBAYASHI Y, TAKAYAMA F, GOMITA Y, KAWASAKI H: Neuronal nitric-oxide synthase inhibition facilitates adrenergic neurotransmission in rat mesenteric resistance arteries. J Pharmacol Exp Ther 316: 490-497, 2006.

HE XR, GREENBERG SG, BRIGGS JP, SCHNERMANN JB: Effect of nitric oxide on renin secretion. II. Studies in the perfused juxtaglomerular apparatus. Am J Physiol 268: F953-F959, 1995.

HUANG CF, HSU CN, CHIEN SJ, LIN YJ, HUANG LT, TAIN YL: Aminoguanidine attenuates hypertension, whereas 7-nitroindazole exacerbates kidney damage in spontaneously hypertensive rats: the role of nitric oxide. Eur J Pharmacol 699: 233-240, 2012.

KRISTEK F: Pentaerythrityl tetranitrate attenuates structural changes in conduit arteries evoked by long-term NO-synthase inhibition. Br J Pharmacol 130: 450-456, 2000. 
KRISTEK F: Some peculiar effects of NO-synthase inhibition on the structure and function of cardiovascular system. Curr Pharm Biotechnol 12: 1305-1315, 2011.

KRISTEK F, GEROVA M: Long-term NO synthase inhibition affects heart weight and geometry of coronary and carotid arteries. Physiol Res 45: 361-367, 1996.

KRISTEK F, GEROVA M, DEVAT L, VARGA I: Remodelling of septal branch of coronary artery and carotid artery in L-NAME treated rats. Physiol Res 45: 329-333, 1996.

KRISTEK F, FABEROVA V, VARGA I: Long-term effect of molsidomine and pentaerythrityl tetranitrate on cardiovascular system of spontaneously hypertensive rats. Physiol Res 52: 709-717, 2003.

KRISTEK F, CACANYIOVA S, GEROVA M: Hypotrophic effect of long-term neuronal no-synthase inhibition on heart and conduit arteries of the Wistar rats. J Physiol Pharmacol 60: 21-27, 2009.

KRISTEK F, MALEKOVA M, ONDRIAS K, CACANYIOVA S: Blood pressure-independent hypotrophy of the heart, kidneys and conduit arteries after 7-nitroindazole administration to Wistar rats from the prenatal period to adulthood. J Physiol Pharmacol 64: 35-39, 2013.

LINARDI A, PANUNTO PC, FERRO ES, HYSLOP S: Peptidase activities in rats treated chronically with N(omega)nitro-L-arginine methyl ester (L-NAME). Biochem Pharmacol 68: 205-214, 2004.

MOORE PK, BABBEDGE RC, WALLACE P, GFFEN ZA, HART SL: 7-Nitro indazole, an inhibitor of nitric oxide synthase, exhibits anti-nociceptive activity in the mouse without increasing blood pressure. $J$ Pharmacol 108: 296-297, 1993.

NAFTILAN AJ: The role of angiotensin II in vascular smooth muscle cell growth. $J$ Cardiovasc Pharmacol 20: S37-S40, 1992.

NGUELEFACK TB, MEKHFI H, DONGMO AB, DIMO T, WATCHO P, ZOHEIR J, LEGSSYER A, KAMANYI A, ZIYYAT A: Hypertensive effects of oral administration of the aqueous extract of Solanum torvum fruits in L-NAME treated rats: Evidence from in vivo and in vitro studies. J Ethnopharmacol 124: 592-599, 2009.

ONO H, ONO Y, FROHLICH ED: Nitric oxide synthase inhibition in spontaneously hypertensive rats: systemic, renal, and glomerular hemodynamics. Hypertension 26: 249-255, 1995.

PALIEGE A, MIZEL D, MEDINA C, PASUMARTHY A, HUANG YG, BACHMANN S, BRIGGS JP, SCHERMANN JB, YANG T: Inhibition of nNOS expression in the macula densa by COX-2-derived prostaglandin E(2). Am J Physiol Renal Physiol 287: F152-F159, 2004.

QADRI F, CARRETERO OA, SCICLI AG: Centrally produced neuronal nitric oxide inthe control of baroreceptor reflex sensitivity and blood pressure in normotensive and spontaneously hypertensive rats. Jpn J Pharmacol 81: 279-285, 1999.

RABELO FA, RUSSO EM, SALGADO MC, COELHO EB: Nonendothelial NO blunts sympathetic response of normotensive rats but not of SHR. Hypertension 38: 565-568, 2001.

SHARMA DK, MANRAL A, SAINI V, SINGH A, SRINIVASAN BP, TIWARI M: Novel diallyldisulfide analogs ameliorate cardiovascular remodeling in rats with L-NAME-induced hypertension. Eur J Pharmacol 691: 198-208, 2012.

THORUP C, PERSSON AEG: Impaired effect of nitric oxide synthesis inhibition on tubuloglomerular feedback in hypertensive rats. Am J Physiol 271: F246-F252, 1996.

TOBA H, KOJIMA Y, WANG JH, NODA K, TIAN W, KOBARA M, NAKATA T: Erythropoietin attenuated vascular dysfunction and inflammation by inhibiting NADPH oxidase-derived superoxide production in nitric oxide synthase-inhibited hypertensive rat aorta. Eur J Pharmacol 691: 190-197, 2012.

TOROK J, KRISTEK F: Beneficial effect of pentaerythrityl tetranitrate on functional and morphological changes in the rat thoracic aorta evoked by long-term nitric oxide synthase inhibition. Vascul Pharmacol 38: 177-182, 2002.

USUI M, EGASHIRA K, TOMITA H, KOYANAGI M, KATOH M, SHIMOKAWA H, TAKEYA M, YOSHIMURA T, MATSUSHIMA K, TAKESHITA A: Important role of local angiotensin II activity mediated via type 1 receptor in the pathogenesis of cardiovascular inflammatory changes induced by chronic blockade of nitric oxide synthesis in rats. Circulation 707: 305-310, 2000.

WEIBEL ER, KISTLER GS, SCHERLE WF: Practical stereological methods for morphometric cytology. J Cell Biol 30: 23-38, 1966. 
WELCH WJ, TOJO A, LEE JU, KANG DG, SCHNACKENBERG CG, WILCOX CS: Nitric oxide synthase in the JGA of the SHR: expression and role in tubuloglomerular feedback. Am J Physiol 277: F130-F138, 1999.

WELCH WJ, TOJO A, WILCOX CS: Roles of NO and oxygen radicals in tubuloglomerular feedback in SHR. Am J Physiol Renal Physiol 278: F769-F776, 2000. 